\title{
The impact of a preloaded intraocular lens delivery system on operating room efficiency in routine cataract surgery
}

This article was published in the following Dove Press journal:

Clinical Ophthalmology

17 June 2016

Number of times this article has been viewed

\author{
Jason J Jones' \\ Jeffrey $\mathrm{Chu}^{2}$ \\ Jacob Graham ${ }^{2}$ \\ Serge Zaluski ${ }^{3}$ \\ Guillermo Rocha ${ }^{4}$ \\ Jones Eye Clinic, Sioux City, IA, \\ ${ }^{2}$ Quorum Consulting Inc., San \\ Francisco, CA, USA; ${ }^{3} \mathrm{VISIS}$, Perpignan, \\ France; ${ }^{4}$ Ocular Microsurgery \& Laser \\ Centre, Brandon, MB, Canada
}

Correspondence: Jason J Jones Jones Eye Clinic, 4405 Hamilton Boulevard, Sioux City, IA 5I I04, USA

Tel + I 7I2 2393937

Fax + I 7|2 239 |305

Email jasonjonesmd@mac.com
Purpose: The aim of this study was to evaluate the operational impact of using preloaded intraocular lens (IOL) delivery systems compared with manually loaded IOL delivery processes during routine cataract surgeries.

Methods: Time and motion data, staff and surgery schedules, and cost accounting reports were collected across three sites located in the US, France, and Canada. Time and motion data were collected for manually loaded IOL processes and preloaded IOL delivery systems over four surgery days. Staff and surgery schedules and cost accounting reports were collected during the 2 months prior and after introduction of the preloaded IOL delivery system.

Results: The study included a total of 154 routine cataract surgeries across all three sites. Of these, 77 surgeries were performed using a preloaded IOL delivery system, and the remaining 77 surgeries were performed using a manual IOL delivery process. Across all three sites, use of the preloaded IOL delivery system significantly decreased mean total case time by $6.2 \%-12.0 \%(P<0.001$ for data from Canada and the US and $P<0.05$ for data from France). Use of the preloaded delivery system also decreased surgeon lens time, surgeon delays, and eliminated lens touches during IOL preparation.

Conclusion: Compared to a manual IOL delivery process, use of a preloaded IOL delivery system for cataract surgery reduced total case time, total surgeon lens time, surgeon delays, and eliminated IOL touches. The time savings provided by the preloaded IOL delivery system provide an opportunity for sites to improve routine cataract surgery throughput without impacting surgeon or staff capacity.

Keywords: time and motion, provider impact, surgical throughput, IOL

\section{Introduction}

Cataract prevalence increases dramatically with age, affecting $\sim 40 \%$ of all adults aged $\geq 70$ years and increasing to $60 \%$ of adults aged $\geq 75$ years. ${ }^{1,2}$ As of 2010 , an estimated 24.4 million American adults had cataracts; due to a substantial aging population, prevalence is expected to double to $>50$ million by $2050 .{ }^{1}$ As the world's population ages, the global prevalence of cataract is likely to increase in the same timeframe, placing an even greater clinical and economic burden on the health care system. With aging populations and a doubling of prevalence, ${ }^{1}$ there is a need for more efficient and effective surgical techniques to provide cataract treatment with reduced resource utilization and costs. ${ }^{3}$

The cost of cataract surgery varies widely depending on location and setting. Data collected from nine European countries showed that in 2005, per surgery costs ranged from $€ 318$ in Hungary to $€ 1,087$ in Italy (or $\sim 344-1,177$ USD). In this 
study, the greatest cost variations were due to overhead and personnel/labor, including the amount of time required for each surgery. ${ }^{3}$ Another research study (2002-2003) found the mean cost of cataract surgery in Europe to be $€ 1,261$ (or $\sim 1,365$ USD). ${ }^{4}$

In cataract surgery, the opacified lens is surgically removed and replaced with an artificial intraocular lens (IOL). The process of handling and manually loading the IOL into an inserter increases surgery duration and presents a chance of contamination. Additionally, although serious complications are uncommon in cataract surgery, this procedure is associated with the greatest number of surgical errors in ophthalmology, including the insertion of an incorrect IOL. ${ }^{5}$ The majority of toxic anterior segment syndrome cases following cataract surgery are attributed to surgical instrument contamination resulting from improper or insufficient cleaning, or to products introduced into the eye during surgery. ${ }^{6}$ Similarly, postoperative endophthalmitis most commonly occurs due to a bacterial infection following cataract surgery and IOL implantation. ${ }^{7}$

Although complications, such as toxic anterior segment syndrome and endophthalmitis, are infrequent, occurring in $0.07 \%$ and $0.04 \%$ of patients receiving cataract surgery respectively, ${ }^{8,9}$ these complications can include severe pain, serious intraocular tissue damage, and dramatic vision loss or blindness, increasing health care utilization and costs. ${ }^{10}$ Exposure of the lens to the surgical environment through a manual loading and insertion process can be a vector for introduction of bacteria that can lead to endophthalmitis. Data from France estimate that hospitalization costs for endophthalmitis are $\sim € 6.36$ million for 1,725 cases ( $€ 3,688$ per patient). ${ }^{11}$ In the US, charges and payments related to treating patients with endophthalmitis are $\sim 2.5$ times higher compared to controls. $^{7}$ Additionally, the risk of complications related to cataract surgery increases with age. One US study found that, compared with patients aged 65-74 years, patients aged 75-84 years had an 11\% higher risk of developing endophthalmitis following cataract surgery, while patients aged $\geq 85$ years had a $53 \%$ higher risk. ${ }^{12}$ It is likely that many of these negative outcomes could be avoided and cost burden alleviated with improved sterile surgical techniques.

In other areas of ophthalmology, intraoperative efficiencies have been achieved with the use of disposable surgical tools. One study found that switching from conventional metal corneal lens rings to disposable sutureless silicone rings during a combined $23 \mathrm{G}$ vitrectomy and cataract procedure resulted in average preparation time savings of 3.94 minutes per procedure. ${ }^{13}$ Use of the disposable sutureless silicone rings also led to significant reductions in intraoperative corneal limbus bleeding and scarring.

As the need for cataract surgery increases with the aging global population, surgeons require improved safety and efficiency in the operating room (OR). ${ }^{3}$ The TECNIS 1-Piece IOL with the TECNIS iTec Preloaded Delivery System (Abbott Medical Optics Inc., Santa Ana, CA, USA) is a notouch preloaded and disposable delivery system designed to allow a cataract surgeon to implant an IOL safely without having to physically touch or manipulate the lens outside of the eye and has the potential to decrease total case time, eliminate IOL handling, and improve throughput.

This study was designed to evaluate the operational impact of converting from a manually loaded IOL process to the single use, no-touch, TECNIS 1-Piece IOL with the TECNIS iTec Preloaded Delivery System in three facilities that perform cataract surgeries.

\section{Methods}

\section{Study settings}

This prospective, observational, multicenter study was conducted at three sites located in the US, France, and Canada. Each site scheduled cataract surgeries $1-2 \mathrm{~d} / \mathrm{wk}$, and each site predominately or exclusively performed routine cataract cases, with a majority $(62 \%-100 \%)$ being monofocal IOL insertions.

In Canada, the participating site was low volume $(\sim 10$ cases/d). The site in France was moderate volume $(\sim 17$ cases $/ d)$, and the site in the US was high volume $(\sim 25$ cases/d), as shown in Table 1. The sites in France and Canada were located within hospital facilities, whereas the

Table I Study site overview

\begin{tabular}{llll}
\hline Site name & $\begin{array}{l}\text { Minnedosa Health } \\
\text { Centre (Canada) }\end{array}$ & $\begin{array}{l}\text { Medipole Ophthalmology } \\
\text { Center (France) }\end{array}$ & Jones Eye Clinic (US) \\
\hline Facility type & Hospital & Hospital & Ambulatory Surgical Center \\
Number of ORs & I & 2 & 2 \\
Cataract schedule (d/wk) & I & I or 2 & 2 \\
Cataract volume & Low $\sim 10$ cases $/ d)$ & Moderate $(\sim 17$ cases $/ d)$ & High $(\sim 25$ cases $/ d)$ \\
Case mix & All routine, monofocal & Predominantly routine & Predominantly routine \\
& cataract cases & cataract cases, 62\% monofocal & cataract cases, 92\% monofocal \\
\hline
\end{tabular}

Abbreviations: ORs, operating rooms; d, day; wk, week. 
US site was located in an Ambulatory Surgical Center. Prior to starting the study, the preloaded IOL system had not been adopted in the Canada and US sites, and the standard of care was for the surgeon to perform the surgery using a manually loaded lens delivery process. In contrast, the site in France already had experience using the preloaded system for routine cataract surgeries. For all study sites, standard practice was for the surgeon to load the IOL themselves. The loading process includes preparing the ophthalmic viscosurgical device-filled cartridge and advancing the plunger to place the lens near the cartridge tip prior to the inserter being placed into the incision.

Each site setup was unique, with specific staffing models designed to support the surgeon and optimize workflows. The France center had two ORs, with one scrub technician (ST) and one circulating nurse $(\mathrm{N})$ in each $\mathrm{OR}$ for the full duration of each surgery day. Two sterilization technicians (CL) cleaned the used equipment and disposables in a cleaning station adjacent to the two ORs. The US site had two ORs and one cleaning station located between the ORs. This site also had three STs rotating between ST and CL roles. Lastly, the center in Canada had one OR with three nurses rotating between ST, N, and surgical preparation nurse roles. The anesthetist remained in the OR during surgery and was responsible for wheeling the patient in and out of the OR. At this center, the cleaning station was located across the hallway from the surgery prep area.

\section{Measures and data collection}

Time-and-motion data, staff and surgery schedules, and cost accounting reports were collected across all three sites. Time and motion data were collected over a total of four surgery days. Staff schedules, surgery schedules, and cost accounting reports were collected in the 2 months prior and after introduction of the preloaded IOL delivery system. In the US site, revenue and claims data were also collected.

The preloaded IOL delivery system utilized throughout the course of the study was the TECNIS 1-Piece IOL with the TECNIS iTec Preloaded Delivery System.

\section{Primary outcome}

The primary outcome was total case time, including OR setup, surgery, and teardown time. OR setup time was defined as the time between when the N or ST opened the ophthalmology pack and when all surgical items were prepped and placed in the sterile field. In France and Canada, surgery began with placement of the speculum by the surgeon and ended with the removal of the speculum or administration of povidoneiodine (Betadine). In the US, surgery began when the surgeon sat in the surgical chair and positioned the microscope. Surgery ended when the surgeon finished checking the status of the eye and provided a verbal indication that surgery was complete. Teardown time was defined as the time following surgery until the N or ST finished applying disinfectant to the surgery table surfaces. To control for variability introduced by nonlens-related workflows, total case time excluded the time between the end of OR setup and start of surgery. This time period varied widely across study sites and included the application of surgical drapes, administration of ophthalmic drops, and other patient-centric activities.

\section{Secondary outcomes}

Secondary outcomes included surgeon lens time, surgeon delays, and the number of IOL touches per case. Surgeon lens time was defined as the length of time the surgeon spent loading, inserting, and positioning the lens in the eye. Surgeon delays were defined as any issues that prevented the surgeon from beginning surgery, such as the staff not having the OR setup complete or the patient was not prepped and ready for surgery. Surgeon delays were collected only in sites with two-OR setups. IOL touches were defined as the number of times that the surgeon physically touched or manipulated the lens while manually loading the inserter.

Time measurement data were collected using UMTPlus ${ }^{\circledR}$ (Laubrass Inc., Montreal, QC, Canada), a time-and-motion work measurement mobile software application. Data recorded for each case included time measurements of the respective tasks performed by the surgeon, ST, N, and CL. The time taken by OR staff to complete key lens-related case components (OR setup time, surgery time, and OR teardown time) was documented and analyzed.

For the Canadian and American sites, time-and-motion measurements were collected over two surgical days prior to and two surgical days after adoption of the preloaded IOL delivery system. In France, because the preloaded system was already in use at the time of study commencement, time-and-motion data using both manual and preloaded IOL delivery systems were collected simultaneously over a 4-day period. All sites collected data from a minimum of 20 cases for each type of lens insertion system, for a minimum of 40 cases at each site. Cases performed with the preloaded IOL delivery system were referred to as "preloaded" cases, and cases performed with a manual IOL system were referred to as "manual" cases. The study was approved by Salus IRB (formerly RCRC IRB). Given that there was no change to patient care (eg, patients received standard of care), the IRB did not require patient written informed consent. 


\section{Data analyses}

Descriptive statistics included the median, minimum, and maximum duration of all time-and-motion metrics (eg, total case, surgery, and surgeon lens times) and IOL touches by individual case at each site. Descriptive statistics, including mean and SD, were also calculated for total case time. Only mean was calculated for IOL touches across sites.

Lens-related delays, nonlens-related delays, and surgeon delays were also recorded. Lens-related delays were delays directly related to the lens, such as lens scratching and loading errors. Lens-related delays were not removed from timeand-motion measurements. Nonlens-related delays were the delays unrelated to the IOL system used for surgery, such as delays due to staff talking. Nonlens-related delays were removed from all time-and-motion measurements. Surgeon delays were recorded separately in the France and US sites (two ORs) and were not removed from time-and-motion measurements.

To compare time-and-motion parameters between manual and preloaded cases, $P$-values were calculated using a Wilcoxon rank sum test for the Canadian center, which had a one-OR setup. General linear models were used to compare outcomes at the sites in France and the US, both of which had a two-OR setup. At the centers with two ORs, data were examined for each OR separately and for both combined.

\section{Results}

\section{Primary outcomes}

The study included a total of 154 routine cataract cases, collected over twelve surgery days across all three sites. Each site reported data from four surgery days. The site in Canada included a total of 40 cases ( 20 preloaded and 20 manual), the site in France included 47 cases (20 preloaded and 27 manual), and the site in the US included 67 cases (37 preloaded and 30 manual). An adoption period of 1-7 months allowed all sites to perform a minimum of 100 monofocal cataract cases using the preloaded IOL delivery system prior to data collection.
At all three sites, use of the preloaded IOL delivery system resulted in significant reductions in mean total case time. In Canada, a $12.0 \%$ reduction in mean total case time was observed, with a mean difference of 3.3 minutes per case $(P<0.001)$; in France, a $6.2 \%$ reduction in mean total case time was observed, with a mean difference of 1.1 minutes per case $(P<0.05)$; and in the US, a 9.4\% reduction in mean total case time was observed, with a mean difference of 2.1 minutes per case $(P<0.001)$. While total case time savings were observed across all sites and segments (OR setup, surgery, and OR teardown), the reduction in time was not statistically significant for all measured time segments across all sites, as shown in Table 2.

\section{Secondary outcomes}

Across all three sites, the preloaded IOL delivery system resulted in significant reductions in surgeon lens times. The site in Canada had a $43.7 \%$ reduction in total surgeon lens time with the preloaded system, with a median difference of 0.8 minutes $(P<0.001)$; the site in France had a $31.9 \%$ reduction with a median difference of 0.4 minutes $(P<0.001)$; and the site in the US had a $17.4 \%$ reduction with a median difference of 0.4 minutes $(P<0.001)$.

Surgeon delays were documented for the two sites that had a 2-OR setup (France and the US), and both found reduced surgeon delays with the preloaded IOL system. In the site in France, surgeon delays occurred in 30\% of cases utilizing a manually loaded process and $20 \%$ of cases utilizing the preloaded system (representing a 33\% reduction), as shown in Table 3. In France, the overall mean duration of surgeon delay was $13 \%$ lower for preloaded IOL cases, at 0.8 minutes, compared to 0.9 minutes with manual cases. In the US site, surgeon delays occurred in $27 \%$ of cases utilizing a manually loaded process and $11 \%$ of cases utilizing the preloaded system (representing a 59\% reduction). The overall mean duration of surgeon delay at this site was $72 \%$ lower for preloaded cases, at 0.3 minutes, compared to 1.1 minutes with manual cases.

Table 2 Reduction in total case time by site

\begin{tabular}{|c|c|c|c|c|c|c|}
\hline & \multicolumn{2}{|c|}{ Canada (one OR) } & \multicolumn{2}{|c|}{ France (two ORs) } & \multicolumn{2}{|c|}{ US (two ORs) } \\
\hline & Diff (\%) & $P$-value & Diff (\%) & $P$-value & Diff (\%) & $P$-value \\
\hline Setup time (median) & -14.4 & $<0.001$ & -7.7 & NS & -17.0 & $<0.001$ \\
\hline Surgery time (median) & -7.8 & $<0.01$ & -8.7 & NS & -3.7 & $<0.10$ \\
\hline Teardown time (median) & -10.3 & NS & -4.1 & NS & -12.5 & $<0.01$ \\
\hline Total case time (mean) & -12.0 & $<0.00$ I & -6.2 & $<0.05$ & -9.4 & $<0.001$ \\
\hline Total case time (SD) & +5.0 & NS & -27.2 & NS & -11.8 & NS \\
\hline
\end{tabular}

Notes: A two-sample $t$-test was used for independent samples. $P$-values above 0.10 were deemed not significant.

Abbreviations: ORs, operating rooms; Diff, difference; NS, not significant; SD, standard deviation. 
Table 3 Surgeon delays in sites with two-OR setups

\begin{tabular}{|c|c|c|c|c|c|c|}
\hline & \multicolumn{3}{|l|}{ France } & \multicolumn{3}{|l|}{ US } \\
\hline & Manual & Preloaded & Diff (\%) & Manual & Preloaded & Diff (\%) \\
\hline Percentage of cases with surgeon delay & 30 & 20 & -33 & 27 & 11 & -59 \\
\hline Mean duration of delay (minutes) & 0.9 & 0.8 & -13 & 1.1 & 0.3 & -72 \\
\hline
\end{tabular}

Abbreviations: OR, operating room; Diff, difference.

The number of IOL touches was recorded only for manual cases, as the preloaded IOL device eliminates the need for IOL touches outside of the eye. For manual cases, the mean number of IOL touches was four at the Canada site and five at both the France and US sites, respectively.

\section{Discussion}

This study was conducted to evaluate the operational impact of converting from a manually loaded IOL delivery process to a preloaded IOL delivery system for routine cataract surgeries. At all study sites, adoption of the preloaded system significantly reduced total case time and total surgeon lens time. Additionally, the incidence and total duration of surgeon delays decreased with the preloaded system. The preloaded delivery system also eliminated the need for the surgeon to handle and manually load the IOL prior to insertion, obviating the likelihood of lens contamination associated with direct handling of the IOL.

The case time efficiencies observed with the preloaded system were attributed to a reduction in serial lens handling and preparation activities. During OR setup, the preloaded system eliminated the assembling of a lens inserter and cartridge, thereby reducing OR setup time between $7.7 \%$ and $17.0 \%$. While the preloaded device requires application of the ophthalmic viscosurgical device, the ST was able to perform this activity in parallel with other surgical steps (eg, phacoemulsification). The surgeon not having to manually load the IOL contributed to a $3.7 \%-8.7 \%$ decrease in total surgery time. In addition, the disposable feature of the preloaded system eliminated the need for the inserter to be placed into the sterilization box and carried into the sterilization area, contributing to a reduction in the OR teardown time of $4.1 \%-12.5 \%$. The combination of these increased efficiencies resulted in an overall decrease in total case time of $6.2 \%-12.0 \%$.

Reductions in total case time and total surgeon time (eg, total surgery time and surgeon delays) have different implications on case throughput depending on the OR setup. In a one-OR setup, switching from a manual IOL-loading process to a preloaded system could allow the site to increase throughput if cumulative time savings achieved during OR setup, surgery, and teardown are equal to or greater than the total time required to perform a single cataract case. In a two-OR setup, the site is optimized to make best use of the surgeons' time, and therefore, switching to a preloaded system could allow an improved throughput if total surgeon time savings are equal to or greater than the time required for the surgeon to start and finish the surgery. Apart from surgery time savings, OR setup and teardown efficiencies provided by a preloaded system only have throughput implications if they reduce the frequency or duration of surgeon delays.

In Canada (one OR), the preloaded system saved an average of 3.4 minutes per surgery case compared to manual loading. During the manual case 2-month economic data collection period, the Canada site averaged 10.2 cases per surgery day with a $100 \%$ monofocal case mix. Assuming no changes are made to surgery volume and case mix, a total of 33.7 minutes could be saved per surgery day by switching from manual lens-loading process to a preloaded system. Adding back the time between the completion of OR setup and surgery start (2.9 minutes) to total case time ( 24.3 minutes) results in a total preloaded case time of 27.2 minutes, which is less than the 33.7 minutes saved throughout the course of the surgery day. As a result, in a low-volume, single OR cataract surgery center, switching from a manual IOL-loading process to a preloaded IOL system saves enough time to add at least one routine cataract case without having to increase staff or surgeon capacity, as shown in Table 4.

In France and the US (two-OR sites), the preloaded system saved a median of 0.6 minutes and 0.4 minutes in surgery

Table 4 Impact of preloaded lens system efficiencies over entire surgery day in a Canadian, one-OR cataract center

\begin{tabular}{ll}
\hline & Canada \\
\hline Mean number of manual cases per surgery day & 10.2 cases/d \\
Monofocal case mix during manual observation period & $100 \%$ \\
Total cataract cases eligible for preloaded system & 10.2 cases/d \\
Mean total case time savings per monofocal cataract case & 3.3 minutes \\
Estimated total case time savings per surgery day & $\mathbf{3 3 . 7}$ minutes \\
Mean time between start of OR setup and completion & $\mathbf{2 7 . 2}$ minutes \\
of OR teardown with preloaded system & \\
Potential increase in cataract throughput without & $\mathbf{I . 2}$ cases \\
\hline increasing surgeon and staff capacity &
\end{tabular}

Abbreviations: $d$, day; OR, operating room. 
Table 5 Impact of preloaded lens system efficiencies over entire surgery day in France and US, two-OR cataract centers

\begin{tabular}{|c|c|c|}
\hline & France & US \\
\hline Mean number of manual cases per surgery day & 17.4 cases/d & 25.2 cases/d \\
\hline Monofocal case mix during manual observation period & $6 \mathrm{I}(\%)$ & $92(\%)$ \\
\hline Total cataract cases eligible for preloaded system & 10.6 cases $/ \mathrm{d}$ & 23.2 cases $/ \mathrm{d}$ \\
\hline Median total surgery time savings per monofocal cataract case & 0.6 (minutes) & 0.4 (minutes) \\
\hline Estimated surgery time savings per surgery day & 5.8 (minutes) & 9.3 (minutes) \\
\hline Median surgeon delay time savings per surgery day & 1.2 (minutes) & 6.3 (minutes) \\
\hline Estimated total surgeon time savings per surgery day & 7.0 (minutes) & I 5.5 (minutes) \\
\hline Median total surgery time with preloaded system & 5.8 (minutes) & 10.4 (minutes) \\
\hline Potential increase in cataract throughput without increasing surgeon and staff capacity & $\begin{array}{l}\text { I.2 (number } \\
\text { of cases) }\end{array}$ & $\begin{array}{l}\text { I.5 (number } \\
\text { of cases) }\end{array}$ \\
\hline
\end{tabular}

Abbreviations: OR, operating room; d, day.

time per monofocal cataract case, respectively. During the manual case 2-month data collection period, the French site averaged 17.4 cases per surgery day with a monofocal case mix of $61 \%$, and US site averaged 25.2 cases per surgery day with a $92 \%$ monofocal case mix, resulting in 5.8 minutes and 9.3 minutes of surgery time savings per surgery day, respectively. In addition, the reduction in both frequency and duration of surgeon delays contributed an additional 1.2 minutes and 6.3 minutes in surgeon time savings per surgery day in France and US, respectively. The reduction in surgeon delays with a preloaded IOL system is believed to be a result of surgery staff being more proactive when handling day-to-day operational variability (eg, retrieving surgical supplies and dealing with uncooperative patients) as a result of efficiencies gained in OR setup and teardown. The total surgeon time savings with a preloaded lens system equates to 7.0 minutes and 15.5 minutes in the France and US sites, which is greater than the median 5.8 minutes and 10.4 minutes required to perform a surgery with a preloaded system, respectively. Similar to the Canadian site (one OR), the preloaded system saves enough surgeon time to add at least one routine cataract case in moderate- and high-volume two-OR surgery centers without impacting surgeon capacity, as shown in Table 5 .

Assuming that each site took advantage of the timeand-motion efficiencies, adding one routine cataract case per surgery day could result in substantial improvements in annual throughput, as shown in Table 6. Assuming that the number of surgery days per month remains unchanged, the low-volume one-OR Canada site could increase annual throughput by $9.9 \%$ or 36 cases, the moderate-volume two-OR France site could increase annual throughput by $5.7 \%$ or 90 cases, and the highvolume two-OR US site could increase annual throughput by $4.0 \%$ or 96 cases.

While the results are promising, this study has some limitations. First, this was a pilot study, and while data were rigorously collected across three sites, it was not powered to demonstrate statistical significance. It was also difficult to prospectively assess the direct impact of case time savings on throughput and profitability due to an inability to control for external variable factors at each site (eg, cataract case mix, seasonality in patient demand, and changes in scheduling). A longer post-adoption observational period may control for such factors.

Second, the estimated improvement in throughput assumes that sites have a broader focus on increasing efficiencies and that time savings achieved with the preloaded system are realized and operationalized by the staff at each site. This analysis does not account for the impact of higher case volume on staff satisfaction and employee morale.

Finally, the study findings are limited by the fact that participating surgeons' standard practice was to load the lens

Table 6 Estimated annual throughput from time efficiencies by site

\begin{tabular}{|c|c|c|c|c|c|c|}
\hline \multirow[b]{2}{*}{ Observation period } & \multicolumn{2}{|c|}{ Canada (one OR) } & \multicolumn{2}{|c|}{ France (two ORs) } & \multicolumn{2}{|c|}{ US (two ORs) } \\
\hline & Manual & Preloaded & Manual & Preloaded & Manual & Preloaded \\
\hline Surgery days/month & 3.0 & 3.0 & 7.5 & 7.5 & 8.0 & 8.0 \\
\hline Mean cases/day & 10.2 & 11.2 & 17.4 & 18.4 & 25.1 & 26.1 \\
\hline Total cases/month & 31 & 34 & $|3|$ & 138 & 201 & 209 \\
\hline Total cases/year & 366 & 402 & $\mathrm{I}, 566$ & 1,656 & $2,4 \mid 2$ & 2,508 \\
\hline Additional annual throughput & \multicolumn{2}{|c|}{$+36(+9.9 \%)$} & \multicolumn{2}{|c|}{+90 (+5.7\%) } & \multicolumn{2}{|c|}{$+96(+4.0 \%)$} \\
\hline
\end{tabular}

Abbreviation: ORs, operating rooms. 
into the inserter themselves (for manual cases). While the observed total surgery time efficiencies may not be directly applicable to sites where surgery staff load the lens into the inserter, use of a preloaded system may result in efficiencies in OR setup and OR teardown.

\section{Conclusion}

This pilot observational study showed that converting from a manually loaded IOL delivery process to a preloaded IOL delivery system for routine cataract surgeries reduces total case time, reduces surgeon lens time, and has the potential to increase case throughput. The preloaded system also eliminates the need for IOL handling outside of the eye, thus reducing the potential for lens contamination and postsurgical complications. For facilities that are focused on improving efficiency, the time savings that can be achieved with the preloaded system may help increase efficiency and effective treatment as the demand for cataract surgery increases.

\section{Acknowledgment}

Quorum Consulting Inc. received funding from Abbott Medical Optics Inc. to conduct the study.

\section{Disclosure}

Jeffrey Chu and Jacob Graham are employees of Quorum Consulting Inc. but do not have a proprietary interest. Facilities in which Jason J Jones and Serge Zaluski worked received honoraria for participating in the study, but neither the doctors nor facilities have a proprietary interest. Guillermo Rocha received an honorarium for prestudy work but has no proprietary interest. The authors report no other conflicts on interest in this work.

\section{References}

1. National Eye Institute [webpage on the Internet]. Cataracts. Available from: www.nei.nih.gov/eyedata/cataract. Accessed March 3, 2015.

2. Acosta R, Hoffmeister L, Román R, Comas M, Castilla M, Castells X. Systematic review of population-based studies of the prevalence of cataracts. Arch Soc Esp Oftalmol. 2006;81(9):509-516.

3. Fattore G, Torbica A. Cost and reimbursement of cataract surgery in Europe: a cross-country comparison. Health Econ. 2008;17(1 suppl): S71-S82.

4. Räsänen P, Krootila K, Sintonen H, et al. Cost-utility of routine cataract surgery. Health Qual Life Outcomes. 2006;4:74.

5. Simon JW, Ngo Y, Khan S, Strogatz D. Surgical confusions in ophthalmology. Arch Ophthalmol. 2007;125:1515-1522.

6. Center for Disease Control. Toxic anterior segment syndrome after cataract surgery - Maine, 2006. MMWR: Morb Mortal Wkly Rep. 2007;56: 629-630.

7. Schmier JK, Halpern MT, Covert DW, Lau EC, Robin AL. Evaluation of Medicare costs of endophthalmitis among patients after cataract surgery. Ophthalmology. 2007;114(6):1094-1099.

8. Hellinger WC, Hasan SA, Bacalis LP, et al. Outbreak of toxic anterior segment syndrome following cataract surgery associated with impurities in autoclave steam moisture. Infect Control Hosp Epidemiol. 2006;27(3):294-298.

9. Lundström M, Barry P, Henry Y, Rosen P, Stenevi U. Evidence-based guidelines for cataract surgery: guidelines based on data in the European Registry of Quality Outcomes for Cataract and Refractive Surgery database. J Cataract Refract Surg. 2012;38(6):1086-1093.

10. Díez R, Jordano L, Hita C [webpage on the Internet]. Toxic anterior segment syndrome (TASS) and prophylaxis against postoperative endophthalmitis. In: Zaidi F, editor. Cataract Surgery. Rijeka: InTech; 2013. Available from: http://www.intechopen.com/books/cataractsurgery/toxic-anterior-segment-syndrome-tass-and-prophylaxisagainst-postoperative-endophthalmitis. Accessed April 22, 2015.

11. Colin X, Berdeaux G, Lafuma A, Salvanet-Bouchara A, Kodjikian L. Inpatient costs of endophthalmitis evaluated for the whole of France. Appl Health Econ Health Policy. 2010;8(1):53-60.

12. Keay L, Gower EW, Cassard SD, Tielsch JM, Schein OD. Postcataract surgery endophthalmitis in the United States: analysis of the complete 2003 to 2004 Medicare database of cataract surgeries. Ophthalmology. 2012;119(5):914-922.

13. Wu JG, Wei RH, Liu AH, Zhou XX, Sun GL, Li XR. Comparison of disposable sutureless silicone ring and traditional metal ring in 23-gauge vitrectomy combined with cataract surgery. Clin Ophthalmol. 2011; 5:901-905.
Clinical Ophthalmology

\section{Publish your work in this journal}

Clinical Ophthalmology is an international, peer-reviewed journal covering all subspecialties within ophthalmology. Key topics include: Optometry; Visual science; Pharmacology and drug therapy in eye diseases; Basic Sciences; Primary and Secondary eye care; Patient Safety and Quality of Care Improvements. This journal is indexed on

\section{Dovepress}

PubMed Central and CAS, and is the official journal of The Society of Clinical Ophthalmology (SCO). The manuscript management system is completely online and includes a very quick and fair peer-review system, which is all easy to use. Visit http://www.dovepress.com/ testimonials.php to read real quotes from published authors. 